\section{RACE, ETHNICITY AND PATIENT-REPORTED OUTCOMES IN CHILDHOOD ONSET SYSTEMIC LUPUS ERYTHEMATOSUS}

1,2R Ezequiel Borgia*, ${ }^{2}$ Matthew J Gurka, ${ }^{2}$ Stephanie L Filipp, ${ }^{3}$ Melissa M Elder, ${ }^{2}$ Michelle I Cardel, ${ }^{4,5}$ Natalie J Shiff NJ, for the CARRA Registry Investigators. ${ }^{1}$ Department of Pediatrics, University of Florida, Gainesville, FL, USA; ${ }^{2}$ Department of Health Outcomes and Biomedical Informatics, University of Florida, Gainesville, FL, USA; ${ }^{3}$ Division of Pediatric Allergy, Immunology and Rheumatology, Department of Pediatrics, University of Florida Gainesville, FL, USA; ${ }^{4}$ University of Saskatchewan, Department of Community Health and Epidemiology, Saskatoon, SK, Canada; ${ }^{5}$ anssen Scientific Affairs, LLC, Horsham, PA

\subsection{6/lupus-2021-lupus21 century.43}

Background The association of race/ethnicity with childhoodonset systemic lupus erythematosus (cSLE) outcomes has been well described, with non-White individuals experiencing a more severe disease phenotype including increased damage accrual and higher rates of renal involvement. However, there are limited data regarding patient-reported outcomes (PROs) across race and ethnic groups in cSLE. This study assesses the association of race/ethnicity with PatientReported Outcomes Measurement Information System (PROMIS ${ }^{\oplus}$ ) in cSLE patients at enrollment in the Childhood Arthritis and Rheumatology Research Alliance (CARRA) Registry.

Methods The CARRA Registry is a convenience cohort of pediatric patients with rheumatic disease including cSLE primarily from North America. This study included cSLE patients enrolled in the CARRA registry within two years from cSLE diagnosis who met $\geq 4 / 11$ ACR classification criteria for lupus. Baseline demographics, laboratory, and disease features as well as PROs were obtained. Analysis of variance (ANOVA) was used to assess the difference in the PROMIS summary Tscore means across races/ethnicities. Relationships between
SLEDAI and PRO scores were analyzed using Pearson's correlation coefficients. Multivariable linear regression analysis was used to examine the association of race and ethnicity with PROMIS scores at baseline. P-values $<0.05$ were considered statistically significant.

Results 588 cSLE patients met inclusion criteria: 84\% were female, 24.2\% non-Hispanic White, 23\% African American, and 19\% Hispanic. The mean age at diagnosis was 13.2 years (SD 3.1). Household income and highest parental education varied by race/ethnic group, as did frequency of rash, leukopenia, and positive anti-Sm antibody (table 1). The cohort had low-moderate baseline disease activity, with a mean SLEDAI of 6.14 (SD 6.8). There was no difference in disease activity across races/ethnicities in one-way ANOVA analysis. The overall PROMIS PHG-7 mean T-score was 38.6 (SD 6.4), more than 1 SD below the general population mean of 50 . The mean Pain Interference T-score of 54 (SD 10) was slightly above, and the Physical Function Mobility mean T-score of 47.5 (SD 10.13) was slightly below the population mean of 50 . There was a negative moderate correlation between PROMIS Pain Interference and Physical Function Mobility (Pearson correlation coefficient: -0.52). There was no association between race/ethnicity and PROMIS scores in multivariable linear regression analysis.

Conclusions In this large multiethnic CARRA pediatric lupus cohort, global health (PGH-7), pain interference, and physical function mobility as measured by PROMIS did not vary across races/ethnicities. The relatively low baseline disease activity may explain this lack of association. Long-term follow-up data is needed to assess for associations between PROs and race/ ethnicity over time.

Abstract 1003 Table 1 Characteristics by race/ethnicity in cSLE patients

\begin{tabular}{|c|c|c|c|c|c|c|c|}
\hline \multicolumn{7}{|c|}{ Features by race/ethnicity } & \multirow[t]{2}{*}{ P-value ${ }^{* *}$} \\
\hline & Total N (\%) & White & African American & Hispanic & Asian & Other or multiracial & \\
\hline Demographics & 588 & & & & & & \\
\hline Female & $492(84.0)$ & $142(24.2)$ & $137(23.0)$ & $109(19.0)$ & $62(11.0)$ & $29(5.0)$ & 0.66 \\
\hline Age diagnosis, mean (SD) & & $13.1(3.0)$ & $13.7(2.8)$ & $12.9(3.5)$ & $12.6(3.2)$ & $12.9(3.4)$ & 0.21 \\
\hline Household income $<25 \mathrm{~K}$ & $75(19.0)$ & $9(2.3)$ & $25(6.4)$ & $31(8.0)$ & $5(1.3)$ & $4(1.0)$ & $<0.001$ \\
\hline No High School ${ }^{1}$ & $49(10.5)$ & $4(1.0)$ & $10(2.1)$ & $27(6.0)$ & $4(1.0)$ & $3(1.0)$ & $<0.001$ \\
\hline \multicolumn{8}{|l|}{ Disease features ${ }^{2}$} \\
\hline Rash & $208(35.4)$ & $71(12.0)$ & $47(8.0)$ & $40(7.0)$ & $35(6.0)$ & $13(2.2)$ & $<0.05$ \\
\hline Arthritis & $319(54.3)$ & $89(15.1)$ & $98(17.0)$ & $68(12.0)$ & $38(6.5)$ & $19(3.2)$ & 0.65 \\
\hline Nephritis & $81(75.0)$ & $20(19.0)$ & $29(27.0)$ & $21(19.0)$ & $7(6.5)$ & $3(3.0)$ & 0.05 \\
\hline Leukopenia & $254(43.2)$ & $59(10.0)$ & $98(17.0)$ & $51(9.0)$ & $31(5.3)$ & $9(2.0)$ & $<0.001$ \\
\hline Anti ds-DNA & $388(71.2)$ & $100(18.4)$ & $108(20.0)$ & $90(17.0)$ & $61(11.2)$ & $19(3.5)$ & 0.09 \\
\hline Anti-Sm & $264(52.2)$ & $56(11.1)$ & $91(18.0)$ & $68(13.4)$ & $37(7.3)$ & $7(1.2)$ & $<0.001$ \\
\hline SLEDAI, mean (SD) & $5.8(6.7)$ & $5.2(6.1)$ & $6.2(7.1)$ & $6.1(7.0)$ & $5.5(6.6)$ & $6.6(6.8)$ & 0.65 \\
\hline \multicolumn{8}{|l|}{$\mathrm{PROs}^{2}$} \\
\hline PROMIS PGH-7, mean T-score (SD) & $38.6(6.2)$ & $37.7(6.1)$ & $38.9(6.9)$ & $39.2(5.7)$ & $39.5(5.9)$ & $37.5(7.3)$ & 0.27 \\
\hline PROMIS Pain, mean T-score (SD) & $53.7(10)$ & $53.3(11)$ & $54.9(10.1)$ & $54.5(9.0)$ & $51(8.8)$ & $53.6(11.7)$ & 0.46 \\
\hline PROMIS Function, mean T-score (SD) & $47.8(10.1)$ & $47.6(10.2)$ & $47.6(10.0)$ & $47.9(11.0)$ & $50.1(9.5)$ & $45.5(10.4)$ & 0.27 \\
\hline
\end{tabular}

